\title{
Pyelonephritis and Bacteremia From Lactobacillus acidophilus
}

\author{
Samad Rasul ${ }^{\mathrm{a}, \mathrm{d}}$, Faria Farhat ${ }^{\mathrm{a}}$, Shweta Ramsahai ${ }^{\mathrm{b}}$, \\ Robert Delapenha ${ }^{a}$, Namrata Shah ${ }^{\mathrm{c}}$
}

\begin{abstract}
Lactobacillus species have been uncommonly reported to cause clinically significant infections in both immunocompetent and immunocompromised hosts. Lactobacillus bacteremia of renal origin remains a rare clinical entity. We describe a case of pyelonephritis and bacteremia from Lactobacillus acidophilus. To our knowledge, this is only the fourth reported case of pyelonephritis caused by Lactobacillus. We report the case of a 63 year old African American female who had a history of right sided nephrolithiasis and hydronephrosis with recent right ureteral stent placement. She was admitted with pyelonephritis and the ureteral stent was subsequently removed. A new right ureteral mass was discovered at the time of stent removal and biopsy revealed high grade urothelial carcinoma. Blood and urine cultures grew Lactobacillus acidophilus. Antibiotic therapy was directed according to susceptibility pattern. Lactobacillus should be considered as a pathogen when it is isolated (in the appropriate clinical setting) from at least two blood cultures or in one blood culture along with another source especially when the host has one or more predisposing conditions. Lactobacillus species are associated with unusual antimicrobial susceptibility patterns. Lactobacillus should be a suspected pathogen in patients with bacteremia due to gram-positive rods whose condition does not improve with vancomycin therapy.
\end{abstract}

Keywords: Lactobacillus; Pyelonephritis; Bacteremia

Manuscript accepted for publication March 13, 2012

${ }^{a}$ Division of Infectious Diseases, Dept. of Internal Medicine, Howard University Hospital, Washington DC, USA

${ }^{\mathrm{b}}$ Division of Infectious Diseases, Department of Internal Medicine, University of Illinois College of Medicine at Peoria, 530 NE Glen Oak Avenue, Peoria, IL 61637, USA

${ }^{\mathrm{c}}$ Dept. of Internal Medicine, Howard University Hospital, Washington DC, USA

${ }^{\mathrm{d}}$ Corresponding author: Samad Rasul. Email: srasul@howard.edu

doi:10.4021/jmc638e

\section{Introduction}

Lactobacillus species are commonly known to colonize the oropharynx, gastrointestinal tract, and female genitourinary tract [1], but they are not part of the normal skin flora $[1,2]$. They are also found in dairy products, certain fruits and vegetables and are employed as probiotics and in manufacturing fermented foods [2-4]. They have been reported, albeit uncommonly, as a cause of clinically significant infections in both immunocompetent and immunocompromised hosts [2]. They are microaerophilic, non-motile and non-spore forming, gram-positive rods which are catalase and oxidase negative $[1,2]$. Lactobacillus species are rarely pathogenic in humans but have been identified in a variety of infections including dental caries, infective endocarditis, vascular graft infections, urinary tract infections, meningitis, chorioamnionitis, endometritis, liver abscess, splenic abscess and bacteremia [5].

Risk factors for developing Lactobacillus infections include neutropenia, broad-spectrum antibiotics, immunosuppressive conditions, acquired immunodeficiency syndrome, malignancy, transplant, selective bowel decontamination and invasive abdominal or respiratory procedures $[1,2,5]$. Infections caused by Lactobacillus may be underreported in the medical literature because of the failure or reluctance to recognize it as a true pathogen [1]. Polymicrobial or mixed infections have been reported in 39\% to $87 \%$ with streptococci, anaerobes, Candida species, enterococci and gram negative bacilli as frequent co-infecting pathogens $[5,6]$. Patients with lactobacillemia diagnosed within 48 hours of admission are more likely to have polymicrobial bacteremia than patients with nosocomial lactobacillemia [5]. We describe a rare case of pyelonephritis caused by Lactobacillus acidophilus in a female patient subsequently diagnosed with high grade urothelial carcinoma.

\section{Case Report}

A 63 year old, African American female presented with fever and right flank pain radiating to her right groin. She first felt 


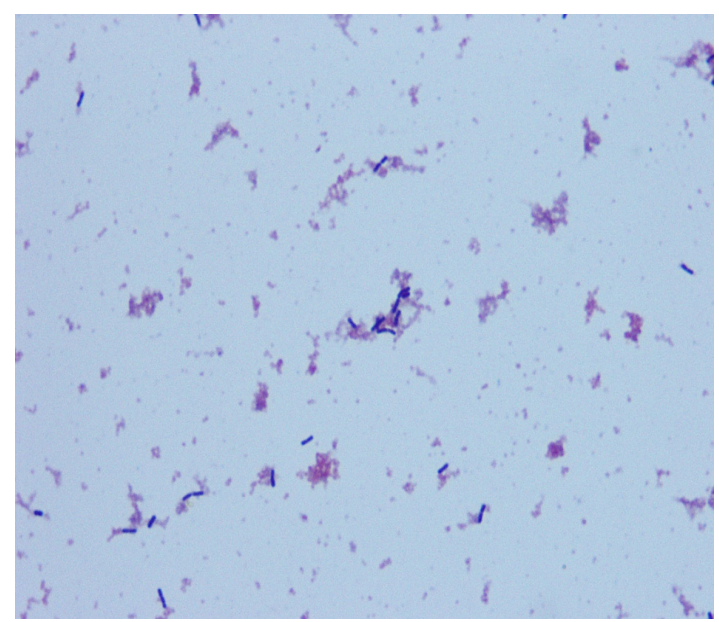

Figure 1. Blood cultures grew non-branching, microaerophilic, non-motile and non-spore forming gram positive bacilli identified as Lactobacillus acidophilus.

these symptoms three days prior to presentation with gradual worsening. Her pain was aggravated by movement and was associated with urinary urgency, rigors and nausea. She noticed that her urine was foul-smelling and appeared cloudy. Her past medical history was significant for uterine cancer status post total abdominal hysterectomy and a remote history of cholecystectomy. She also had a history of severe allergy to penicillin. Four months ago, she developed rightsided obstructive urolithiasis with hydronephrosis requiring placement of a right lower ureteral stent. She received a two week course of levofloxacin after stent placement.

On admission she had a temperature of $38.2{ }^{\circ} \mathrm{C}$, with a pulse rate of 105 beats per minute and blood pressure of $134 / 68 \mathrm{mmHg}$. Physical examination revealed right flank and costovertebral angle tenderness without signs of peritonitis. Laboratory tests showed the following: leukocyte count of 16,500 cells $/ \mathrm{ml}$ ( $89.6 \%$ neutrophils, $12 \%$ bands) and hemoglobin of $11.8 \mathrm{~g} / \mathrm{dl}$. Urinalysis revealed numerous leukocytes, 5 to 9 red blood cells with a positive leukocyte esterase reaction and was nitrite negative. Intravenous ciprofloxacin was initiated empirically after obtaining blood cultures. Computerized tomography scan of her abdomen and pelvis revealed mild worsening of right hydronephrosis with increased perinephric stranding. Renal flow studies were consistent with right-sided hydronephrosis.

The following day, the patient underwent cystoscopic removal of the right ureteral stent, which was found to have visible calcifications. A new mass was discovered in the lower part of the ureter and was biopsied. A higher right ureteral stent was placed to relieve current obstructive hydronephrosis. Urine culture revealed $>10^{5}$ colony forming units $/ \mathrm{ml}$ of Lactobacillus species. Two blood cultures obtained initially on admission subsequently grew gram positive bacilli later identified as Lactobacillus acidophilus (Fig. 1). Intravenous clindamycin was added to ciprofloxacin. The patient's condition improved and she was discharged from the hospital on clindamycin and ciprofloxacin with a follow-up appointment in the urology clinic. Preliminary blood cultures at the time of discharge were negative, but one out of two blood cultures was later noted to grow L. acidophilus. Tumor biopsy results subsequently revealed high grade urothelial carcinoma.

\section{Discussion}

Lactobacillus bacteremia of renal origin is rarely described in the medical literature. In a review of 55 patients with $\mathrm{Lac}$ tobacillus bacteremia, only 6 patients had underlying urinary tract infection as a clinical manifestation [2, 5]. Furthermore, pyelonephritis from Lactobacillus is even more rarely reported. We found only 3 reported cases of Lactobacillus bacteremia with pyelonephritis [7-9]. The first case described pyelonephritis in a patient with nephrolithiasis, who underwent nephrectomy and antibiotic treatment with subsequent full recovery [7]. The second report described emphysematous pyelonephritis in a diabetic patient with dialysis-dependent end stage renal disease and also had a history of breast cancer and liver cirrhosis. This patient was managed with antibiotics and a nephrectomy was performed. However, the patient died from sepsis and complications from multiple comorbidities [8]. A third report described pyelonephritis in a female patient with a history of follicular lymphoma of the breast and type 2 diabetes mellitus. She was found to have hydronephrosis from a stone in the left distal ureter. She responded to antibiotic therapy and did not require surgical removal of her left kidney [9].

Our patient had significant underlying risks for developing Lactobacillus bacteremia and pyelonephritis. Her remote history of uterine cancer and subsequent diagnosis of urothelial cancer rendered her immunocompromised. In addition, she had a history of obstructive hydronephrosis with urological manipulation and placement of stent (foreign device) four months prior to her admission. She received antibiotics on a previous admission, conceivably another risk factor for Lactobacillus sepsis. Since Lactobacillus is a normal commensal bacterium of the urinary system, compromise to the integrity of the ureter could have placed her at significant risk for bacteremia and sepsis. The combination of her clinical presentation of sepsis and pyelonephritis, with three blood cultures and one urine culture positive for Lactobacillus acidophilus (and lack of any other growth) was our criteria for diagnosis of Lactobacillus bacteremia rather than contamination of blood cultures from genitourinary flora or transient mucosal tissue-based bacteremia. To our knowledge, this is the first reported case of Lactobacillus bacteremia and pyelonephritis in a patient with a primary urological malignancy. 
Lactobacillus species are associated with unusual antimicrobial susceptibility patterns and several investigators have reported vancomycin resistance in Lactobacillus [4, 6, 10]. The frequent use of vancomycin in hospitalized patients might explain this pathogenic feature of Lactobacillus in this patient population [1]. Early literature recommends the use of combination therapy with penicillin or another $\beta$-lactam agent with an aminoglycoside for synergy $[1,2]$. In view of our patient's severe allergy to penicillin, this approach was not possible. A review of 85 cases of Lactobacillus bacteremia showed that antimicrobial susceptibility is species-dependent [10]. All isolates had low minimum inhibitory concentrations (MICs) of imipenem, piperacillin-tazobactam, erythromycin and clindamycin. MICs of vancomycin were $>256 \mu \mathrm{g} / \mathrm{mL}$ for all species except Lactobacillus gasseri, Lactobacillus jensenii and Lactobacillus acidophilus which tend to have lower MICs $[1,5,10]$. Lactobacilli are variably resistant to the cephalosporins and quinolones, which are therefore not recommended [10]. Most reports recommend combination therapy based on susceptibility results, especially for endocarditis and other deep-seated infections $[1,5,10]$.

Given the unusual and variable sensitivities, susceptibility testing is of utmost clinical importance [10]. Most automated systems do not identify Lactobacillus at a species level $[2,3,6]$. This might jeopardize outcomes due to species specific variations in antibiotic susceptibility. Molecular analysis of the 16S ribosomal RNA gene is an accurate method of species identification $[3,10]$. The mechanism of vancomycin resistance is not fully known but might be due to altered peptide sequences leading to weakened vancomycin binding, or antibiotic exclusion from the target sites by the cell wall [2]. Some have also reported the possibility of plasmid-mediated resistance. Mortality varies from $26 \%$ to $44 \%$ in different reports, but is generally accepted to be due to rapidly fatal underlying or co-morbid conditions rather than the direct virulence of Lactobacillus $[5,10]$.

Lactobacillus should be a suspected pathogen in patients with bacteremia due to gram-positive bacilli whose condition does not improve with vancomycin therapy [1]. Currently, there is no gold standard for susceptibility testing of Lactobacillus isolates and no guidelines for interpreting test results [10]. Erythromycin, penicillin, clindamycin, aminoglycosides, and imipenem are considered antibiotics of choice and combination therapy has been noted in most studies $[1,5,10]$. Lactobacillus should be considered as a pathogen when it is isolated (in the appropriate clinical setting) from at least two blood cultures or in one blood culture along with another source of infection, especially when the host has one of the predisposing conditions mentioned above [2]. It must also be appreciated that although direct mortality from Lactobacillus bacteremia might be low, its occurrence might indicate the presence of an ominous underlying condition and thus poor long term prognosis $[5,10]$.

\section{Financial Disclosure}

The authors solemnly declare that they have no financial disclosures. This manuscript is not being considered for publication elsewhere.

\section{References}

1. Antony SJ, Stratton CW, Dummer JS. Lactobacillus bacteremia: description of the clinical course in adult patients without endocarditis. Clinical infectious diseases : an official publication of the Infectious Diseases Society of America. 1996;23(4):773-778.

2. Antony SJ. Lactobacillemia: an emerging cause of infection in both the immunocompromised and the immunocompetent host. Journal of the National Medical Association. 2000;92(2):83-86.

3. Tommasi C, Equitani F, Masala M, Ballardini M, Favaro M, Meledandri M, Fontana C, et al. Diagnostic difficulties of Lactobacillus casei bacteraemia in immunocompetent patients: a case report. Journal of medical case reports. 2008;2:315.

4. Russo A, Angeletti S, Lorino G, Venditti C, Falcone M, Dicuonzo G, Venditti M. A case of Lactobacillus casei bacteraemia associated with aortic dissection: is there a link? The new microbiologica. 2010;33(2):175-178.

5. Husni RN, Gordon SM, Washington JA, Longworth DL. Lactobacillus bacteremia and endocarditis: review of 45 cases. Clinical infectious diseases : an official publication of the Infectious Diseases Society of America. 1997;25(5):1048-1055.

6. Salminen MK, Rautelin H, Tynkkynen S, Poussa T, Saxelin M, Valtonen V, Jarvinen A. Lactobacillus bacteremia, clinical significance, and patient outcome, with special focus on probiotic L. rhamnosus GG. Clinical infectious diseases : an official publication of the Infectious Diseases Society of America. 2004;38(1):62-69.

7. Manzella JP, Harootunian R. Lactobacillemia of renal origin: a case report. The Journal of urology. 1982;128(1):110.

8. Morgan M, Hunter LK. Lactobacillus sepsis and emphysematous pyelonephritis. Infections in Medicine 2004; 21:79-82.

9. Chazan B, Raz R, Shental Y, Sprecher H, Colodner R. Bacteremia and pyelonephritis caused by Lactobacillus jensenii in a patient with urolithiasis. The Israel Medical Association journal : IMAJ. 2008;10(2):164-165.

10. Salminen MK, Rautelin H, Tynkkynen S, Poussa T, Saxelin M, Valtonen V, Jarvinen A. Lactobacillus bacteremia, species identification, and antimicrobial susceptibility of 85 blood isolates. Clinical infectious diseases : an official publication of the Infectious Diseases Society of America. 2006;42(5):e35-44. 